\title{
Quantum phase transitions in Bose-Einstein condensates from a Bethe ansatz perspective
}

\author{
D. Rubeni ${ }^{\text {a }}$, A. Foerster ${ }^{\text {a,* }}$, E. Mattei ${ }^{a}$, I. Roditi $^{b}$ \\ a Instituto de Física da UFRGS, Porto Alegre, RS, Brazil \\ ${ }^{\mathrm{b}}$ Centro Brasileiro de Pesquisas Físicas - CBPF/MCTI, 22290-180, Rio de Janeiro, RJ, Brazil
}

Received 5 October 2011; received in revised form 23 November 2011; accepted 28 November 2011

Available online 1 December 2011

\begin{abstract}
We investigate two solvable models for Bose-Einstein condensates and extract physical information by studying the structure of the solutions of their Bethe ansatz equations. A careful observation of these solutions for the ground state of both models, as we vary some parameters of the Hamiltonian, suggests a connection between the behavior of the roots of the Bethe ansatz equations and the physical behavior of the models. Then, by the use of standard techniques for approaching quantum phase transition - gap, entanglement and fidelity - we find that the change in the scenery in the roots of the Bethe ansatz equations is directly related to a quantum phase transition, thus providing an alternative method for its detection.
\end{abstract}

(c) 2011 Elsevier B.V. All rights reserved.

Keywords: Quantum phase transitions; Bose-Einstein condensation; Integrable models; Bethe ansatz

\section{Introduction}

The study of Bose-Einstein condensates has provided in recent years several new exciting possibilities of research, either experimental or theoretical. One of these is the investigation of different phases of ultracold atoms. Whether, for instance, one can take them from an insulator to a superfluid behavior, as in [1]. Now, given the fact that these condensates reside in the realm of near to absolute zero temperatures new tools are needed to approach this quantum phase transition. One possibility lies in the field of quantum information and make use of quantities such as the entanglement entropy or the fidelity. Another possibility that we would like to advance

\footnotetext{
* Corresponding author. Tel.: +55 513166471 ; fax: +55 513191762.

E-mail address: angela@if.ufrgs.br (A. Foerster).
} 
is the use of quantum integrable systems which contributed with several models of Bose-Einstein condensates [2-7] where one has exact control over their solutions through Bethe ansatz methods and whose importance has been highlighted in [8,9].

Quantum phase transitions most distinctive feature, when compared with a classical phase transition, is that they take place at zero temperature. That is, in practical terms, when quantum fluctuations are more relevant than thermal ones. This is reflected in the behavior of the ground state, of the investigated physical system, which changes in some essential manner when some parameter of the associated Hamiltonian takes different values. The typical image is presented in [10] for a Hamiltonian given by a linear combination of commuting operators, when changing a coefficient of the combination, the ground state may reach a non-analytic point for some critical value of this coefficient.

For quantum integrable systems one can have access to the ground state through the exact solution of the corresponding models by the quantum inverse scattering method that leads to the algebraic Bethe ansatz equations [11-14]. A careful observation of the behavior of solutions of these equations for the ground state, as we vary some parameters of the Hamiltonian, suggests a connection between the behavior of roots of the Bethe ansatz equations and the physical behavior of such models. Which is exactly what we expect to happen in quantum phase transitions.

Our aim is then, for two different integrable models, the two-site Bose-Hubbard model and the hetero-atomic molecular Bose-Einstein condensate (BEC), to compare our results, coming from the study of the solutions of the Bethe ansatz equations, with the results of other more standard methods for finding quantum phase transitions such as the study of the entanglement, energy gap and fidelity. Notwithstanding that rigorously a quantum phase transition is only defined in the limit where $N$, the number of particles in the system, approaches infinity, the previously mentioned concepts show a clear response to variations in the ground state of the system for finite $N$ and unequivocally indicate the presence of quantum phase transitions [15]. Very recent experiments incorporating the realization of a quantum system exhibiting classical bifurcations as well as the determination of a suitable criteria for measuring the entanglement between two wells in a BEC [16-18] turn this kind of investigation for such models even more appealing.

In the next section we analyze the attractive two-site Bose-Hubbard model where a bifurcation in its classical analysis indicates the possibility of a quantum phase transition; we investigate the structure of the ground state distribution of roots of the Bethe ansatz equations for this model in order to examine if its behavior could be used to unveil a quantum phase transition. A comparison is done with the behavior of such quantities as the entanglement, energy gap and fidelity. We also provide an image of which processes occur in the phase transition. In the third section we apply the same kind of analysis to the hetero-atomic molecular Bose-Einstein condensate, which is described by a three mode Hamiltonian that allows for atomic and molecular states to exist as a superposition. In Section 4 we summarize our results and draw some conclusions.

\section{Attractive two-site Bose-Hubbard model}

From the theoretical point of view, the two-site Bose-Hubbard model, also known as the canonical Josephson Hamiltonian [2,19], has been a useful model in understanding tunneling and self-trapping phenomena. The Hamiltonian is given by

$$
\hat{H}=\frac{k}{8}\left(\hat{N}_{1}-\hat{N}_{2}\right)^{2}-\frac{\mu}{2}\left(\hat{N}_{1}-\hat{N}_{2}\right)-\frac{\epsilon}{2}\left(\hat{a}_{1}^{\dagger} \hat{a}_{2}+\hat{a}_{2}^{\dagger} \hat{a}_{1}\right),
$$


where $\left\{\hat{a}_{j}^{\dagger}, \hat{a}_{j} \mid j=1,2\right\}$ are the creation and annihilation operators for the condensate $j$, associated respectively to two bosonic Heisenberg algebras, with the following commutation relations

$$
\left[\hat{a}_{i}, \hat{a}_{j}^{\dagger}\right]=\delta_{i j}, \quad\left[\hat{a}_{i}, \hat{a}_{j}\right]=\left[\hat{a}_{i}^{\dagger}, \hat{a}_{j}^{\dagger}\right]=0
$$

also the operators $\hat{N}_{j}=\hat{a}_{j}^{\dagger} \hat{a}_{j}$ are the corresponding boson number operators for each condensate. Since the Hamiltonian does not depend explicitly on time and commutes with the total boson number $\hat{N}=\hat{N}_{1}+\hat{N}_{2}$, the total number of bosons $N$ is a conserved quantity and it is possible to set ourselves to a subspace with fixed value of $N$. The coupling $k$ provides the strength of the scattering interaction between bosons and may be attractive $(k<0)$ or repulsive $(k>0)$. The parameter $\mu$ is the external potential which corresponds to an asymmetry between the condensates and $\epsilon$ is the coupling for the tunneling. Despite its apparent simplicity, this model exhibits very interesting behavior. In particular, an investigation of its quantum dynamics predicts nontrivial threshold couplings [20,21], in qualitative agreement with experimental results [22]. We restrict ourselves to study the case $k<0$ because it is known that in the attractive case the system presents a quantum phase transition (QPT) [21,23].

\subsection{Classical analysis}

Let $\left\{\hat{N}_{j}, \hat{\theta}_{j} \mid j=1,2\right\}$ be quantum variables satisfying the canonical commutation relations. In order to go to the classical limit it is convenient to make a change of variables from the operators $\left\{\hat{a}_{j}^{\dagger}, \hat{a}_{j} \mid j=1,2\right\}$ to a number-phase representation via

$$
\hat{a}_{j}=e^{i \hat{\theta}_{j}} \sqrt{\hat{N}_{j}}, \quad \hat{a}_{j}^{\dagger}=\sqrt{\hat{N}_{j}} e^{-i \hat{\theta}_{j}}
$$

such that the Heisenberg canonical commutation relations are preserved. Now we define the variables

$$
\hat{z}=\frac{1}{\hat{N}}\left(\hat{N}_{1}-\hat{N}_{2}\right)
$$

representing the fractional occupation imbalance and

$$
\hat{\theta}=\frac{\hat{N}}{2}\left(\hat{\theta}_{1}-\hat{\theta}_{2}\right)
$$

representing the phase difference. Note that $(\hat{z}, \hat{\theta})$ are canonically conjugate variables. In the classical limit where $N$ is large, but still finite, we may equivalently consider the Hamiltonian $[2,24]$

$$
H(z, \theta)=\frac{\epsilon N}{2}\left[\frac{\lambda}{2} z^{2}-\beta z-\sqrt{1-z^{2}} \cos \left(\frac{2 \theta}{N}\right)\right],
$$

where $\lambda=\frac{k N}{2 \epsilon}, \beta=\frac{\mu}{\epsilon}$ are the parameters governing the different dynamic regimes of the condensates atomic tunneling. The Hamilton's equations of motion of the system are given by

$$
\begin{aligned}
& \dot{\theta}=-\frac{\partial H}{\partial z}=\frac{\epsilon N}{2}\left[\lambda z-\beta+\frac{z}{\sqrt{1-z^{2}}} \cos \left(\frac{2 \theta}{N}\right)\right], \\
& \dot{z}=\frac{\partial H}{\partial \theta}=-\epsilon\left[\sqrt{1-z^{2}} \sin \left(\frac{2 \theta}{N}\right)\right] .
\end{aligned}
$$




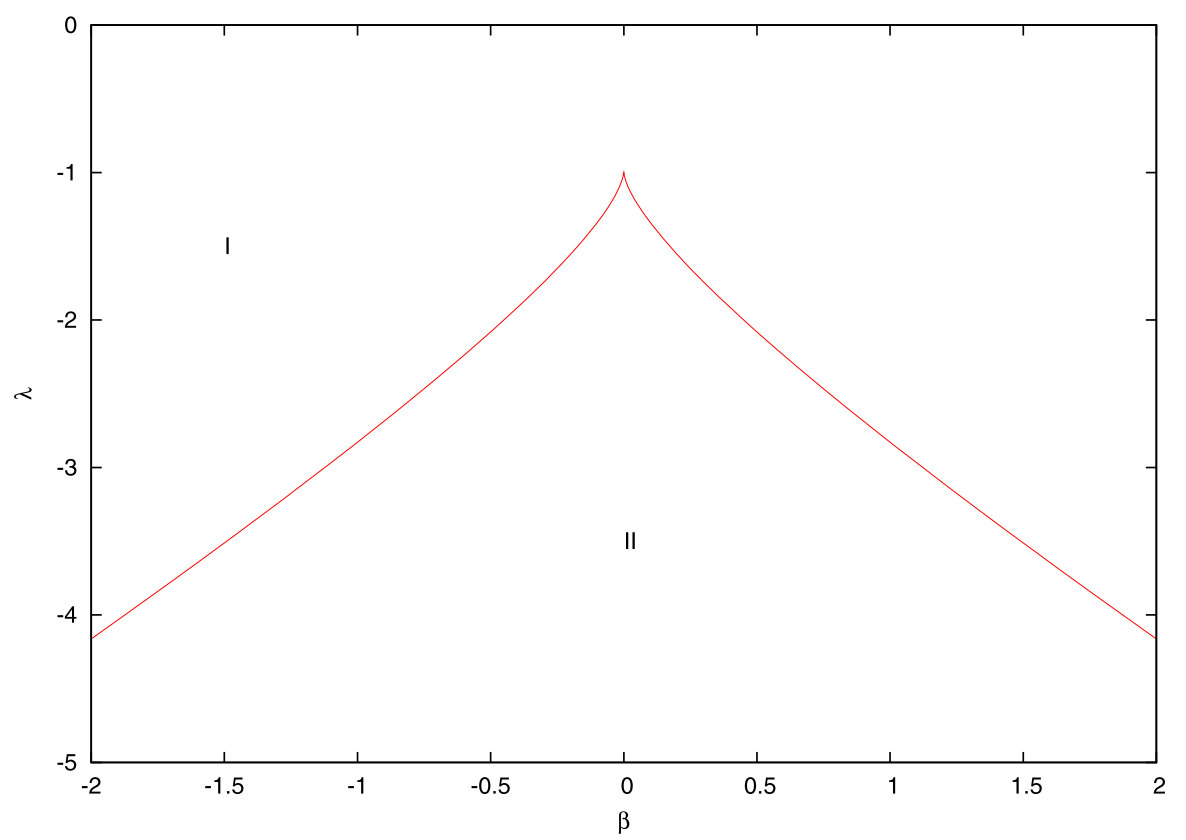

Fig. 2.1. Coupling parameter space diagram identifying the different types of solutions for the equations of fixed points $\dot{z}=\dot{\theta}=0$. In the region I there is one solution for $\theta=\frac{N \pi}{2}$ and one solution for $\theta=0$ and in region II there is one solution for $\theta=\frac{N \pi}{2}$ and three solutions for $\theta=0$.

Here we would like to mention that the above classical equations of motion were also derived in [24]. Now we study the fixed points of the Hamiltonian (2.6), determined from the above equations by the condition $\dot{z}=\dot{\theta}=0$. By performing a numerical analysis we find that these equations may have one, two or three solutions, depending on the values of the coupling parameters (see [25] for details). This allow us to construct a diagram of parameters identifying the different types of solutions, depicted in Fig. 2.1. We remark that in the absence of the external potential $(\beta=0)$, we have a fixed point bifurcation given by $\lambda_{0}=-1$.

It has been conjectured $[26,27]$ that fixed points in the classical analysis can be used to identify quantum phase transitions in a general level, regardless of the nature of the bifurcation. This model exhibits a bifurcation point in the parameter diagram and becomes a natural candidate to study quantum phase transitions.

\subsection{Bethe ansatz solution}

The two-site Bose-Hubbard model has a very special property, it is an exactly solvable model. The integrability of this model was demonstrated using the Quantum Inverse Scattering Method $($ QISM) [2]. A major advantage of the QISM, or algebraic Bethe ansatz method is that it leads to an exact solution for the integrable models, furnishing the energy eigenvalues and the Bethe ansatz equations (BAE) for the models. The set of BAE and energies for the Hamiltonian (2.1) can be written in two different ways: (i) in a product form as well as (ii) in an additive form [28]. Here we will employ the additive form, given by [28]

$$
E=\frac{k N^{2}}{8}-\frac{\mu N}{2}+\frac{\epsilon}{2} \sum_{j=1}^{N} v_{j},
$$


where the parameters $v_{j}$ must satisfy the following BAE:

$$
\frac{\epsilon v_{j}^{2}+[k(1-N)-2 \mu] v_{j}-\epsilon}{k v_{j}^{2}}=\sum_{k \neq j}^{N} \frac{2}{v_{k}-v_{j}} .
$$

Thus, each set $\left\{v_{j}, j=1, \ldots, N\right\}$ solution of BAE (2.10), provides an energy eigenvalue (2.9) of the Hamiltonian. In principle, it is not realizable the analytical solution of these equations. There are, however, a few studies in asymptotic limits for the repulsive case [29]. Nevertheless due to their structure, in order to achieve a more accurate analysis of the model, we may employ numerical tools. The numerical solution of Eq. (2.10), shows that the ground state in the attractive case has the structure of a $N$-string - i.e., it presents itself as a collection of symmetric solutions in the complex plane like $\left\{v_{j} \mid j=1, \ldots, N\right\}=\left\{x_{k} \pm i y_{k} \mid k=1, \ldots, \frac{N}{2}\right\}$ - in contrast with the repulsive case, where the ground-state solution has always real roots. All these solutions have been checked with the exact diagonalization of the Hamiltonian and there is a full agreement. In the following charts we show the solutions of the BAE for some values of the total number of particles and we adopted $\mu=0$ by the consideration of two main reasons: (i) non-zero values of $\mu$ do not significantly alter the behavior of the system, just shifting the energy levels [21]; (ii) much of the experimental realizations with these systems are made on the condition of zero external potential [16]. For further analysis, it is useful to take into account the parameter $\lambda^{-1}$, borrowed from the classical analysis:

$$
\lambda^{-1}=\frac{2 \epsilon}{k N} .
$$

In Fig. 2.2, notice the abrupt change in the distribution of roots of Eq. (2.10) in the complex plane from a certain critical value $\lambda_{C}^{-1}$. For values of $\lambda^{-1}$ larger then $\lambda_{C}^{-1}$ the roots begin to distribute about distinct families of curves. The same characteristic behavior of the ground state roots of the Bethe ansatz equations is observed for other values of $N$ - see Figs. 2.3 and 2.4.

The largest value of $N$ for which we have numerically solved the BAE (2.10) and to determine the configurations of the roots into the ground state was $N=120$, as illustrated in Fig. 2.4. It is interesting to note that equations similar to (2.10) were found and solved for other integrable models of interest such as the reduced BCS model [30] and the BCS wave-p type model [31], where it was possible to solve the BAE for $N=100$ and $N=30$ Cooper pairs, respectively.

Later we will discuss this peculiar behavior of the solutions of BAE (2.10) under the light of the physical phenomena occurring in the system.

\subsection{Quantum phase transitions}

As discussed in the classical analysis, the fixed points can be used to identify quantum phase transitions in a general level. The two-site Bose-Hubbard model has a bifurcation point that can be seen in the parameters diagram presented in Fig. 2.1 and becomes a natural candidate for the study of entanglement and quantum phase transitions. Quantum Phase Transitions (QPT) occur at absolute zero temperature due to quantum fluctuations when we vary some parameter [10]. Although QPT are rigorously defined only in the thermodynamic limit, when $N$ goes to infinity, the analysis made in finite systems, using the techniques below, indicates points that show a response to variations in the ground state and indicates the presence of quantum phase transitions [15]. Then in order to simplify the nomenclature we use the terminology QPT also when referring to these points. There are different techniques to identify them and, in particular, the 


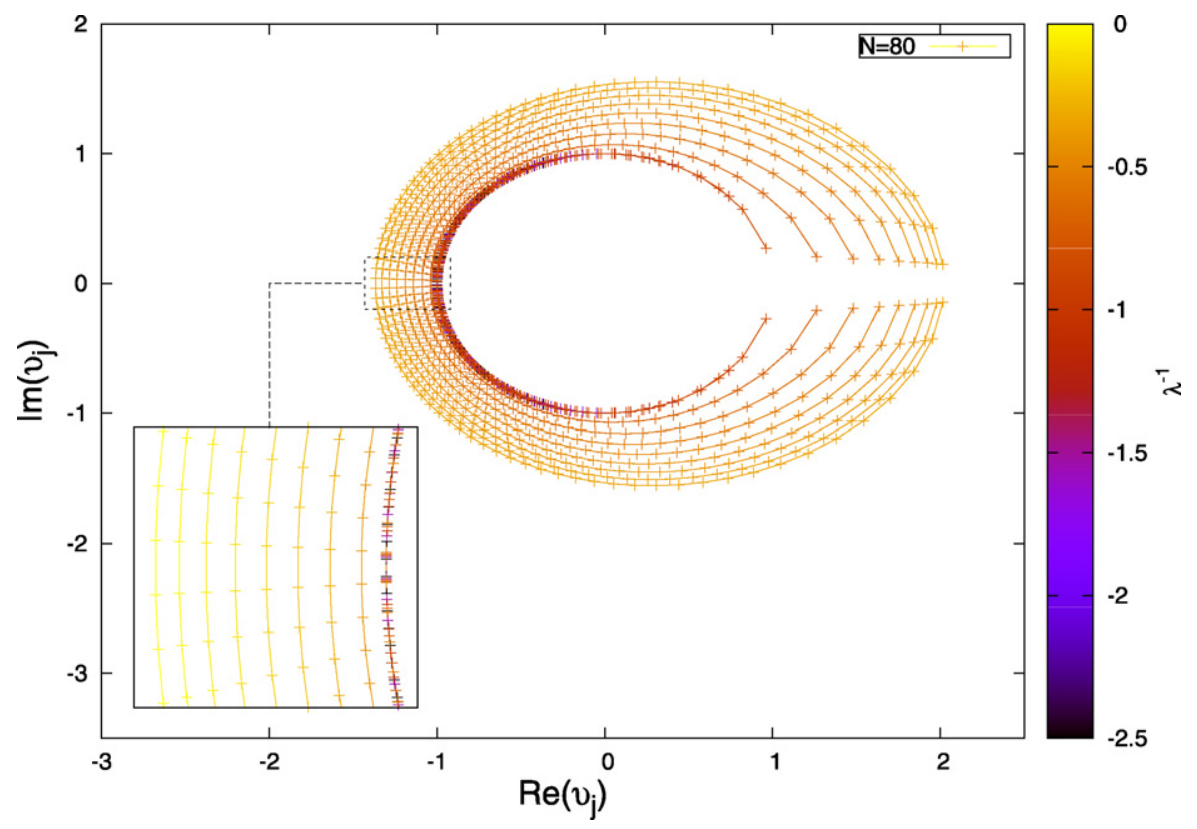

Fig. 2.2. Solutions of Bethe ansatz equations (2.10) in the complex plane for the ground state considering the particular case $N=80$. Each curve corresponds to a value of the parameter $\lambda^{-1}$ as the color table, right, and sits on a set of points (roots of Eq. (2.10)). Here, and in the subsequent figures, the inset shows an enlarged view of the delimited area. The abrupt change in the distribution of roots occurs at $\lambda^{-1} \approx-0.833$.

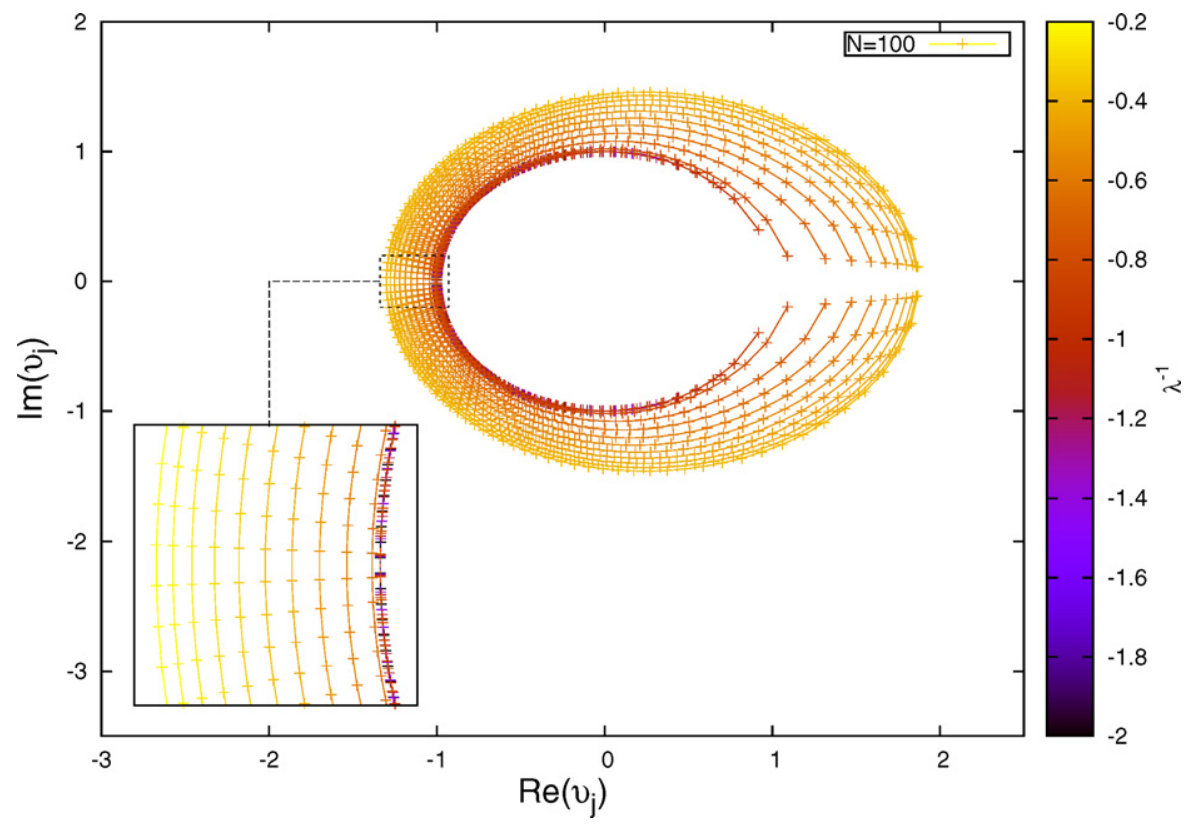

Fig. 2.3. Solutions of Bethe ansatz equations (2.10) in the complex plane for the ground state considering the particular case $N=100$. Each curve corresponds to a value of the parameter $\lambda^{-1}$ as the color table, right, and sits on a set of points (roots of Eq. (2.10)). The abrupt change in the distribution of roots occurs at $\lambda^{-1} \approx-0.909$. 


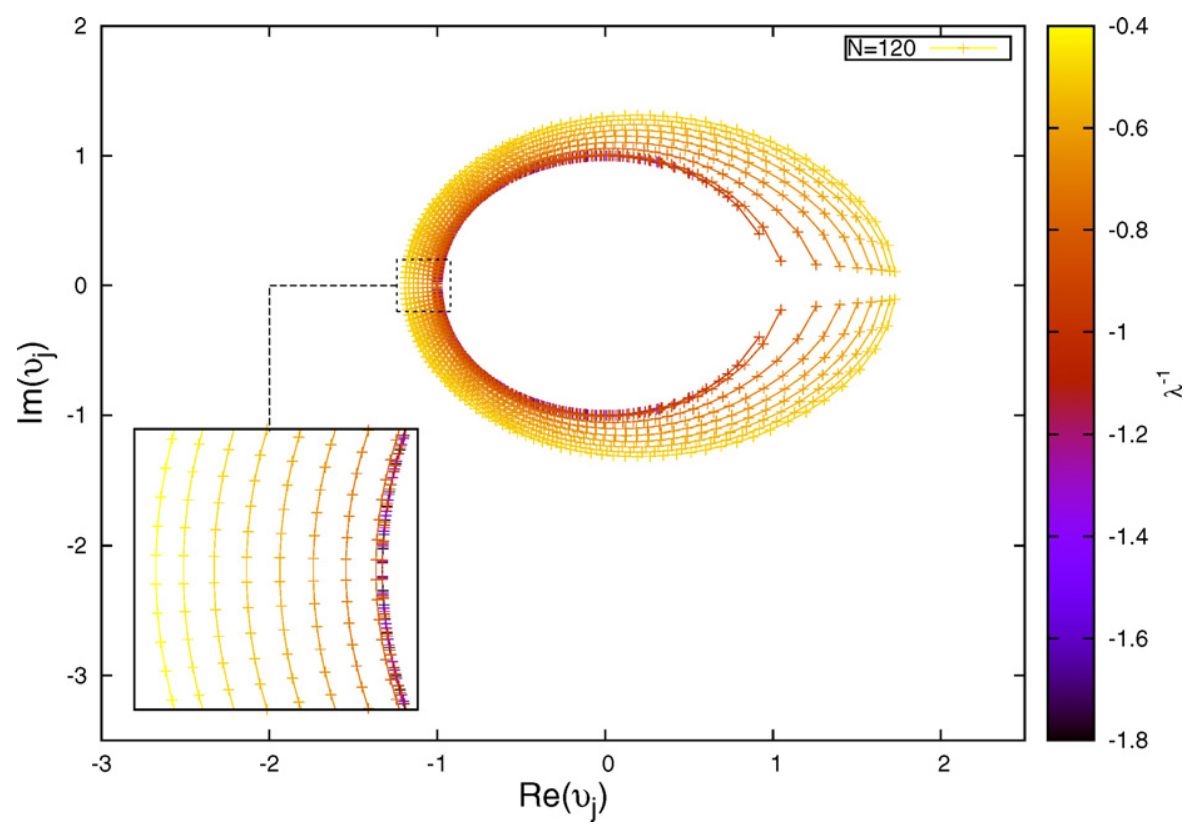

Fig. 2.4. Solutions of Bethe ansatz equations (2.10) in the complex plane for the ground state considering the particular case $N=120$. Each curve corresponds to a value of the parameter $\lambda^{-1}$ as the color table, right, and sits on a set of points (roots of Eq. (2.10)). The abrupt change in the distribution of roots occurs at $\lambda^{-1} \approx-0.926$.

critical values of the parameters for which the transitions happen. We will use three different ones, all of them sensible to the ground state behavior, entanglement, energy gap and fidelity.

\subsubsection{Entanglement}

We can consider the pair of coupled Bose-Einstein condensates as a bipartite system of two modes, "1" and " 2 ". In this case, the standard measure of entanglement is the von Neumann entropy of the reduced density operator of either of the modes [20]. The state of each mode is characterized by its occupation number. Using the fact that the total number of atoms $N$ is constant, a general state of the system can be written in terms of the Fock states by

$$
|\Psi\rangle=\sum_{n=0}^{N} c_{n}|n\rangle|N-n\rangle
$$

where $c_{n}$ are complex numbers and for $N_{1}=n$ atoms in condensate 1 there will be $N_{2}=N-n$ atoms in condensate 2 . The density operator of the system is given by

$$
\rho=|\Psi\rangle\left\langle\Psi\left|=\sum_{m, n=0}^{N} c_{m}^{*} c_{n}\right| m\right\rangle|N-m\rangle\langle n|\langle N-n| .
$$

Taking the partial trace with respect to mode " 2 " yields the reduced density operator for mode " 1 ",

$$
\rho_{1}=\operatorname{Tr}_{2}(\rho)=\sum_{n=0}^{N}\left|c_{n}\right|^{2}|n\rangle\langle n| .
$$




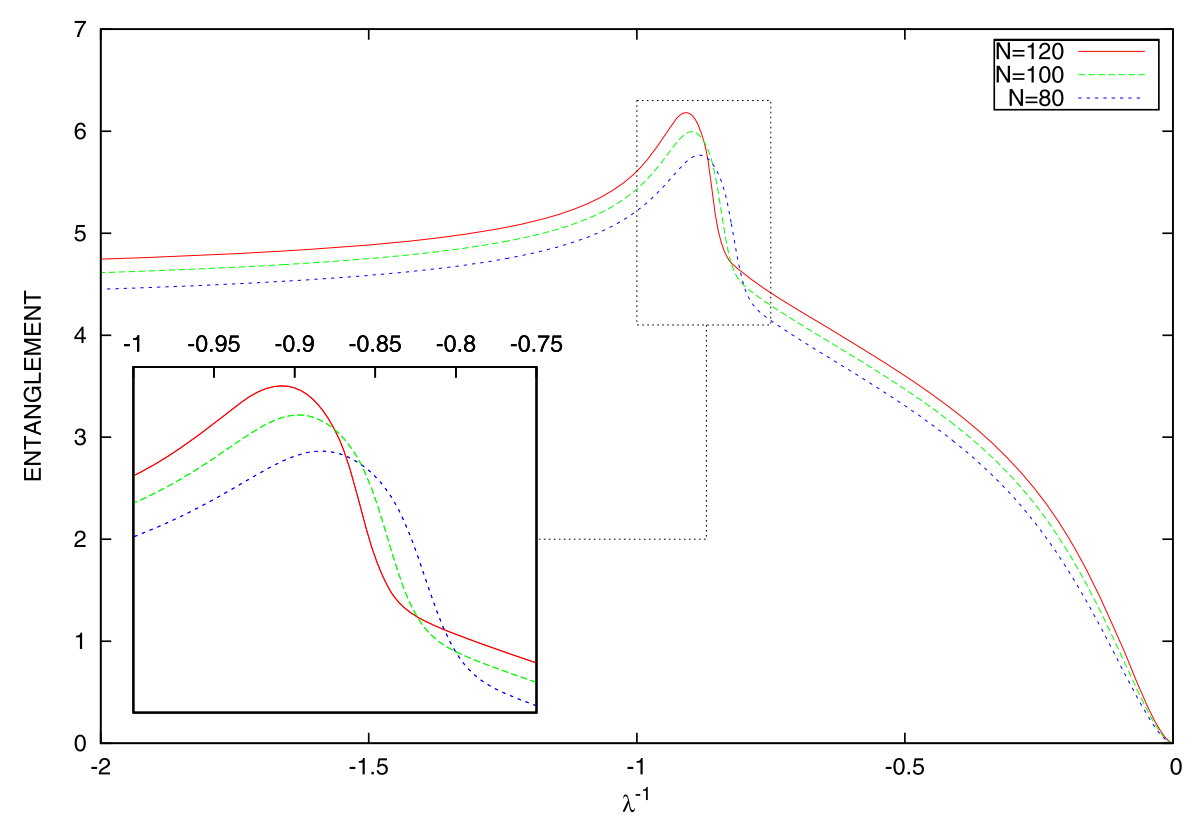

Fig. 2.5. Entanglement entropy of the ground state as a function of $\lambda^{-1}$ for different values of the total number of atoms $N$. The maximum of the entanglement, indicating the QPT point, occurs at $\lambda^{-1}=-0.88,-0.91,-0.93$ for $N=80,100,120$, respectively. As $N$ increases the maximum entropy moves to the critical point $\lambda_{C}^{-1}=-1$.

Thus the entropy of entanglement of the ground-state of the system is given by

$$
E\left(\rho_{1}\right)=-\operatorname{Tr}\left[\rho_{1} \log \left(\rho_{1}\right)\right]=-\sum_{n=0}^{N}\left|c_{n}\right|^{2} \log \left(\left|c_{n}\right|^{2}\right) .
$$

The value of the parameter for which the entropy of entanglement has a maximum identifies the parameters of the QPT [32], depicted in Fig. 2.5. We fix the parameter $\epsilon=1$ and use $k$ as the negative variable coupling parameter.

\subsubsection{Energy gap}

We consider now the energy gap, which is defined as the difference between the first excited state and the ground-state of the system,

$$
\Delta E=E^{(1)}-E^{(0)} \text {. }
$$

The value of the parameter for which the gap is zero, or has a minimum, identifies the parameters of the QPT [10]. In Fig. 2.6 we present the energy gap as a function of $\lambda^{-1}$ for $N=80,100$ and 120.

\subsubsection{Fidelity}

Another possibility to investigate the QPT is through the behavior of the fidelity, which is a concept widely used in the Quantum Information Theory [33]. The fidelity is basically defined as the modulus of the wave-function overlap between two states

$$
\mathcal{F}(\psi, \phi)=|\langle\psi \mid \phi\rangle| \text {. }
$$




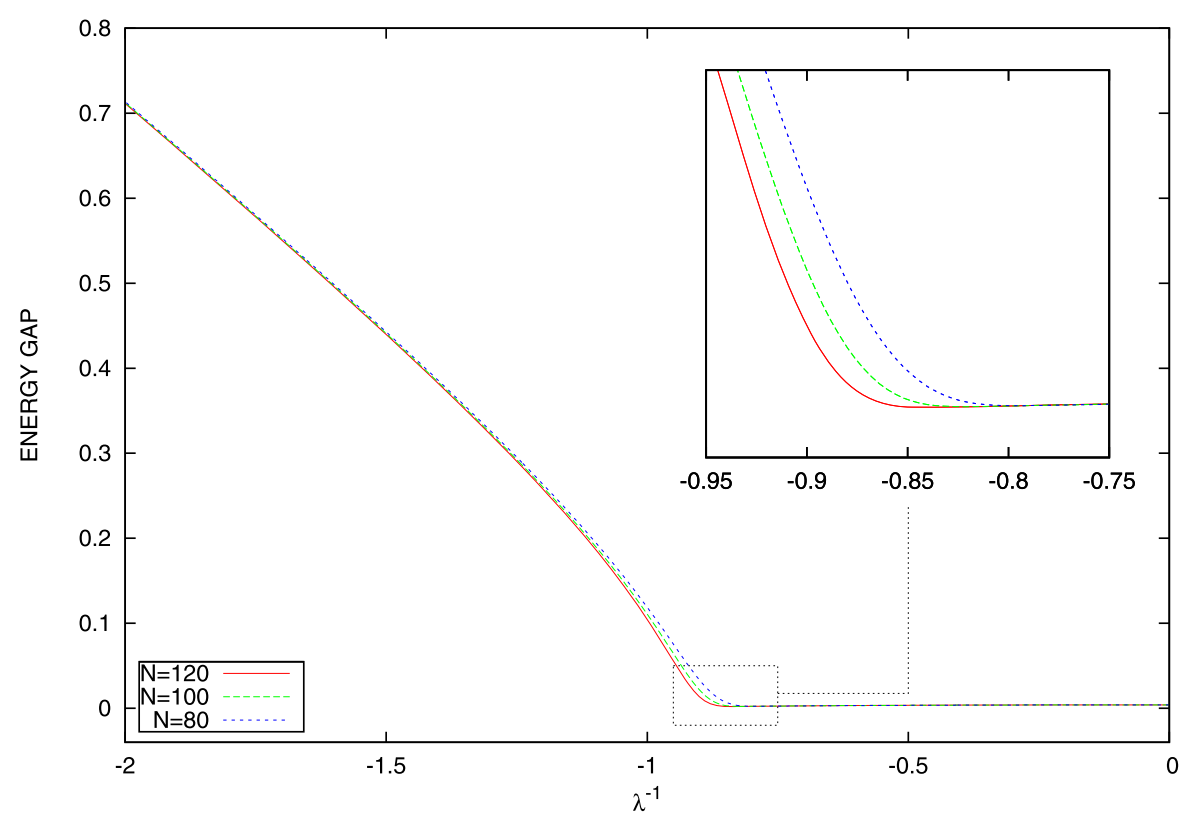

Fig. 2.6. Energy gap of the ground state as a function of $\lambda^{-1}$ for different values of the total number of atoms $N$. The minimum of the energy gap, indicating the QPT point, occurs at $\lambda^{-1}=-0.80,-0.83,-0.85$ for $N=80,100,120$, respectively. As $N$ increases the minimum of the energy gap moves to the critical point $\lambda_{C}^{-1}=-1$.

Basically, the point where the fidelity has a sharp decline defines a critical value for the parameter [34].

\subsection{BAE and $Q P T$}

Observing the graphs - Figs. 2.5, 2.6 and 2.7 - we identify the critical points of QPT for a given set of parameters and we note the striking correspondence between the points obtained by investigating the entanglement, energy gap and fidelity, that indicate a QPT, and the point where the respective ground state solutions of the BAE change their behavior. We also note that, as the number of particles increases, the correlations between the transition point predicted by standard methods and the one predicted by the change of behavior of solutions of BAE agrees with greater precision and in all cases it tends the value $\lambda_{C}^{-1}=-1$, as predicted by the classical analysis. Therefore the behavior of the ground state of the model translated as the behavior of the set of solutions of the BAE, when the relevant parameters are changed, can be used as an alternative method to locate the points of the phase transition of the system.

We interpret this QPT through the behavior of the expected value of the normalized number of atoms in the condensates. In Fig. 2.8 we show the ground state expectation value of the normalized number of particles in the condensates 1 and 2 , for $N=120$ particles. We observe that for low values of $\lambda^{-1}$, the expected value of the normalized number of atoms in the condensates 1 and 2 is $\frac{\left\langle N_{1}\right\rangle}{N} \cong 0.5$ and $\frac{\left\langle N_{2}\right\rangle}{N} \cong 0.5$, respectively, meaning that approximately half of the atoms is in the condensate 1 and the other half is in the condensate 2 . By varying the parameter $\lambda^{-1}$ we find that this value suffers a sharp drop near the critical point $\lambda_{C}=-1$, approaching the values $\frac{\left\langle N_{1}\right\rangle}{N} \cong 1.0$ and $\frac{\left\langle N_{2}\right\rangle}{N} \cong 0.0$, respectively, after crossing this point. So we can interpret this 


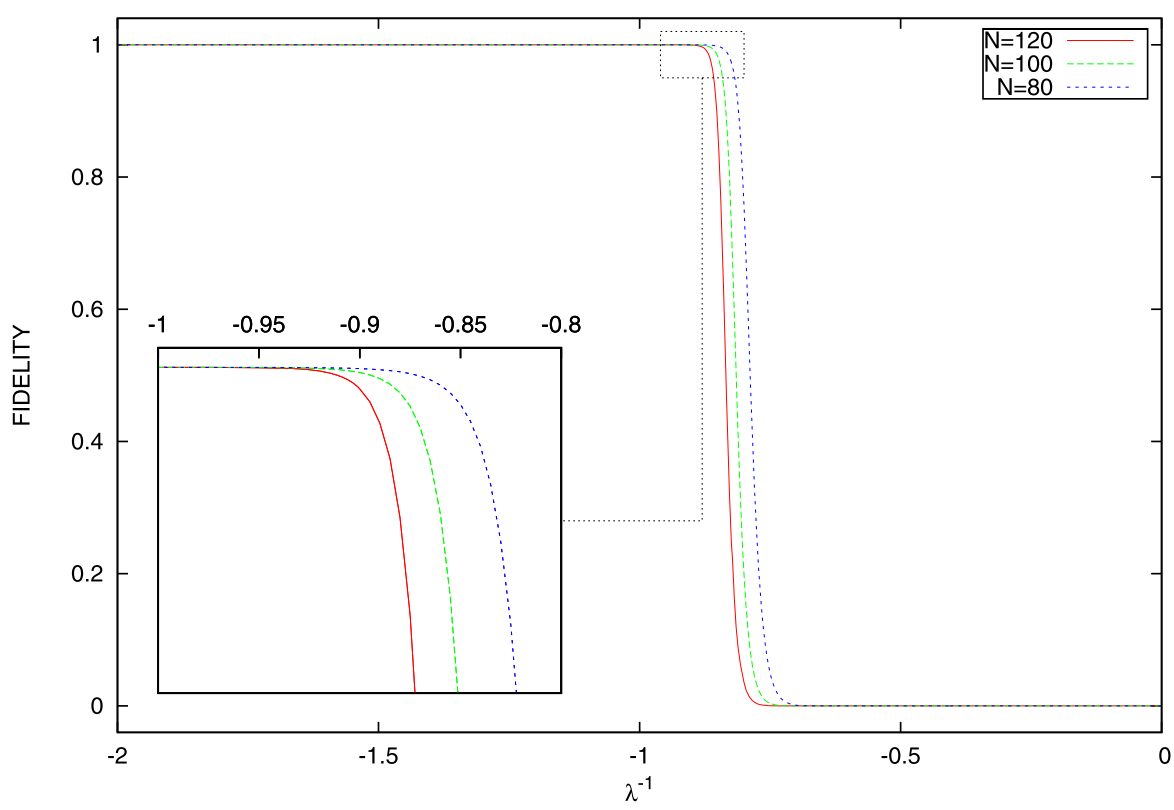

Fig. 2.7. Fidelity of the ground state as a function of $\lambda^{-1}$ for different values of the total number of atoms $N$. The value at which the fidelity has an abrupt decay, indicating the QPT point, occurs at $\lambda^{-1}=-0.88,-0.91,-0.93$ for $N=80$, 100,120 , respectively. As $N$ increases the sharp decline moves to the critical point $\lambda_{C}^{-1}=-1$.

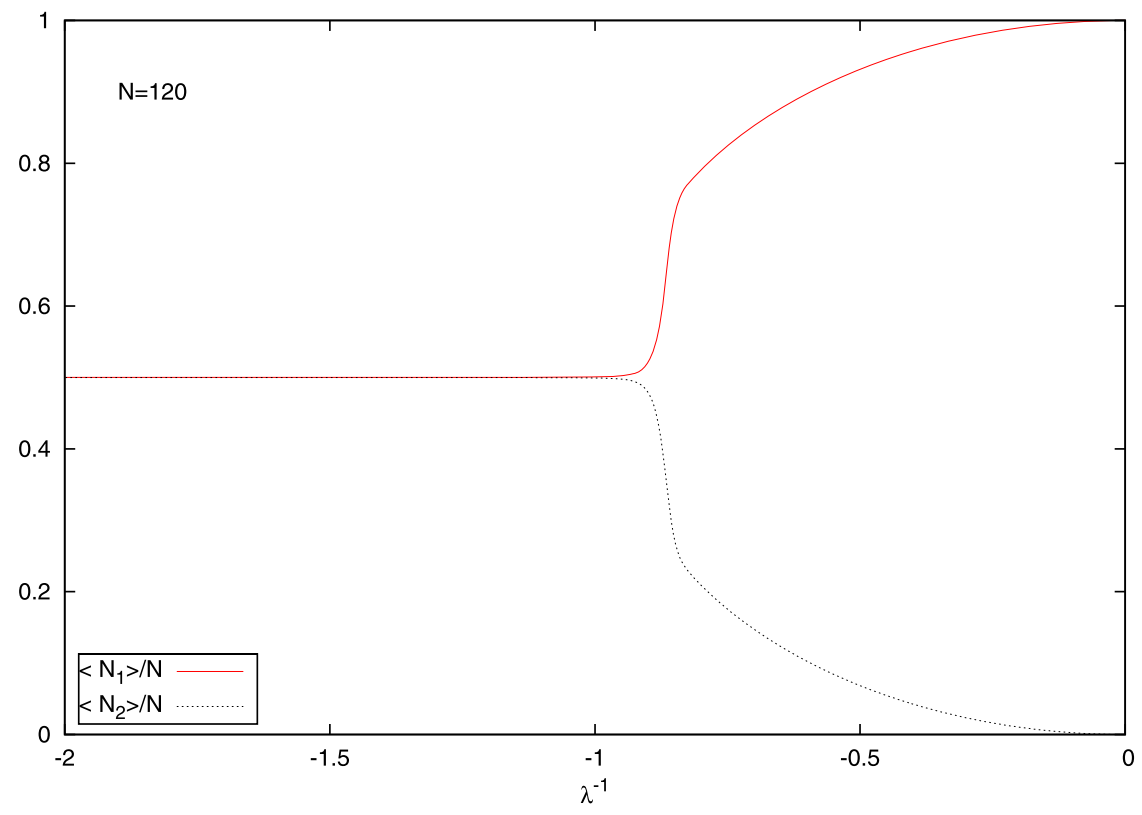

Fig. 2.8. Ground state expectation values for the fraction of the number of atoms in the condensates 1 and 2 as a function of $\lambda^{-1}$. We can observe an abrupt change in the behavior near the critical point $\lambda_{C}^{-1}=-1$. 
transition as the separation between a delocalized phase, with tunneling of atoms between two condensates, and a localized phase.

\section{Hetero-atomic molecular Bose-Einstein condensate}

We now consider a model for two distinct species of atoms that form a hetero-nuclear molecular Bose-Einstein condensate and is described by a three mode Hamiltonian. A novel feature of a molecular Bose-Einstein condensate is that atomic and molecular states can exist as a superposition [35]. For the cases where the molecules are heteronuclear, the presence of a permanent electric dipole moment also opens the possibility for manipulating the condensate through electrostatic forces [36]. These characteristics make the study of such models increasingly interesting allowing to access phenomena that goes from novel quantum phase transitions to ultracold chemistry [37]. The Hamiltonian reads

$$
\hat{H}=\mu_{c} \hat{N}_{c}+\Omega\left(\hat{a}^{\dagger} \hat{b}^{\dagger} \hat{c}+\hat{c}^{\dagger} \hat{a} \hat{b}\right)
$$

where we label each atomic species by $a$ and $b$, which can combine to produce a molecule labeled by $c$. This is the Hamiltonian studied in [38] and also known as the optical Hamiltonian. Again $\left\{\hat{j}, \hat{j}^{\dagger} \mid j=a, b, c\right\}$ are the canonical creation and annihilation operators satisfying the usual bosonic Heisenberg commutation relations of Eq. (2.2) and $\hat{N}_{j}=\hat{j}^{\dagger} \hat{j}$ are the corresponding boson number operators. The parameter $\mu_{c}$ is the external potential and $\Omega$ is the amplitude for interconversion of atoms and molecules. The Hamiltonian commutes with $\hat{J}=\hat{N}_{a}-\hat{N}_{b}$ and with the total number $\hat{N}=\hat{N}_{a}+\hat{N}_{b}+2 \hat{N}_{c}$. We refer to $\hat{J}$ as the atomic imbalance and introduce $k=\frac{J}{N}, k \in[-1,1]$, as the fractional atomic imbalance. A discussion of the physical implications of the imbalance can be found, for example, in [39-41].

\subsection{Classical analysis}

Let $\left\{\hat{N}_{j}, \hat{\theta}_{j} \mid j=a, b, c\right\}$ be quantum variables satisfying the canonical commutation relations

$$
\left[\hat{N}_{j}, \hat{\theta}_{k}\right]=i \delta_{j k} I, \quad\left[\hat{N}_{j}, \hat{N}_{k}\right]=\left[\hat{\theta}_{j}, \hat{\theta}_{k}\right]=0 .
$$

As in Section 2.1 we make a change of variables from the operators $\hat{j}^{\dagger}, \hat{j}$ to a number-phase representation via

$$
\hat{j}=e^{i \hat{\theta}_{j}} \sqrt{\hat{N}_{j}}, \quad \hat{j}^{\dagger}=\sqrt{\hat{N}_{j}} e^{-i \hat{\theta}_{j}},
$$

such that the Heisenberg canonical commutation relations are preserved. Now we define the canonically conjugate variables $\hat{z}$ and $\hat{\theta}$ as

$$
\begin{aligned}
& \hat{z}=\frac{1}{\hat{N}}\left(\hat{N}_{a}+\hat{N}_{b}-2 \hat{N}_{c}\right), \\
& \hat{\theta}=\frac{\hat{N}}{4}\left(\hat{\theta}_{a}+\hat{\theta}_{b}-\hat{\theta}_{c}\right) .
\end{aligned}
$$

In the limit of large $N$ we can obtain from the optical Hamiltonian the approximate (rescaled) classical Hamiltonian

$$
H(z, \theta)=2 \alpha(z-1)+\sqrt{2(1-z)\left(z+c_{+}\right)\left(z+c_{-}\right)} \cos \left(\frac{4 \theta}{N}\right),
$$


where $\alpha=-\frac{\mu_{c}}{\Omega \sqrt{2 N}}$, and $c_{ \pm}=1 \pm 2 k$. The Hamilton's equations of motion are given by

$$
\begin{aligned}
& \dot{\theta}=-\frac{\partial H}{\partial z}=2 \alpha+\frac{2(1-z)(1+z)-\left(z+c_{+}\right)\left(z-c_{-}\right)}{\sqrt{2(1-z)\left(z+c_{+}\right)\left(z+c_{-}\right)}} \cos \left(\frac{4 \theta}{N}\right), \\
& \dot{z}=\frac{\partial H}{\partial \theta}=-\frac{4}{N} \sqrt{2(1-z)\left(z+c_{+}\right)\left(z+c_{-}\right)} \sin \left(\frac{2 \theta}{N}\right) .
\end{aligned}
$$

From these equations, the fixed points of the system can be determined by the condition $\dot{z}=$ $\dot{\theta}=0$. It was shown in [39] that this model exhibits a QPT just in the case of zero imbalance, i.e., $k=0$. For this reason we deal here only with this case, for which it was demonstrated that there are two fixed points $\alpha=1$ and $\alpha=-1$, associated to a minimum and to a maximum in the parameter phase diagram, respectively [39]. Therefore, for the quantized system, it is expected that $\alpha=1$ may indicate a QPT.

\subsection{Bethe ansatz solution}

The Hamiltonian (3.1) is also an integrable model [2]. The energy eigenvalues of the heteroatomic molecular Bose-Einstein condensates are given by

$$
E=-\Omega \sum_{j=1}^{(N-J) / 2} v_{j}
$$

where the parameters $v_{j}$ must satisfy the following Bethe ansatz equations (BAE):

$$
\frac{J+1}{v_{j}}-v_{j}-\frac{\mu}{\Omega}=\sum_{k \neq j}^{(N-J) / 2} \frac{2}{v_{k}-v_{j}} .
$$

The numerical solution of Eq. (3.10) shows that the ground state has the structure of real roots solely. All these solutions have been checked with the exact diagonalization of the Hamiltonian and there is a full agreement.

Notice the abrupt change in the distribution of roots of Eq. (3.10) from a certain critical value $\alpha_{C}$, shown in Fig. 3.1. For values of $\alpha$ smaller than $\alpha_{C}$ the roots are tightly packed when close to the origin and we have a distribution beginning exactly at the origin. By increasing the value of the parameter $\alpha$ the density of roots are not so dense at the origin and tend to get distanced from it. This process reaches its culmination in $\alpha_{C}$ approximately 1 , where no roots are at zero. After $\alpha_{C}$ the set of roots moves away from the origin. Therefore, a different behavior of the BAE-solutions when we cross the region $\alpha<1$ to the region $\alpha>1$ is observed. For other values of $N$, such as $N=80$ and $N=100$, a very similar behavior of the ground state BA-roots is also obtained. In the case of non-zero imbalance $(k \neq 0)$, all roots are away from the origin, independent of the value of $\alpha$. In this case, where it is known that there is no QPT, we also do not observe a different behavior in the solution of the BAE.

\subsection{Quantum phase transitions}

Here as in Section 2.3 we will use three different techniques to identify quantum phase transitions: entanglement, energy gap and fidelity. We use the same definitions as in Eqs. (2.15)-(2.17) and the results are shown in the next Figs. 3.2-3.4. 


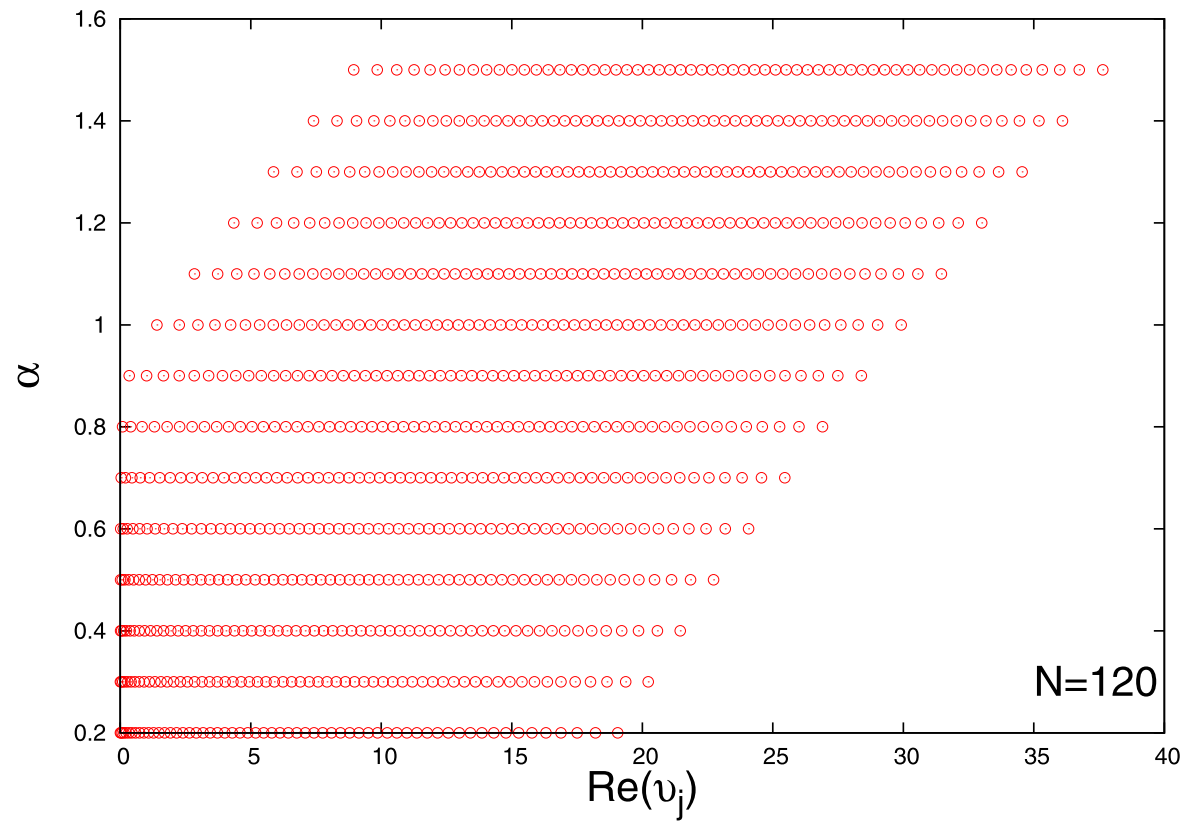

Fig. 3.1. Solutions of Bethe ansatz equations (3.10) for the ground state versus $\alpha$ considering the case $N=120$. Each line corresponds to a BA-solution for a particular value of the parameter $\alpha$. There is a change in the behavior of the distribution of the BA-roots around the critical value $\alpha_{C}=1$.

\section{Entanglement}

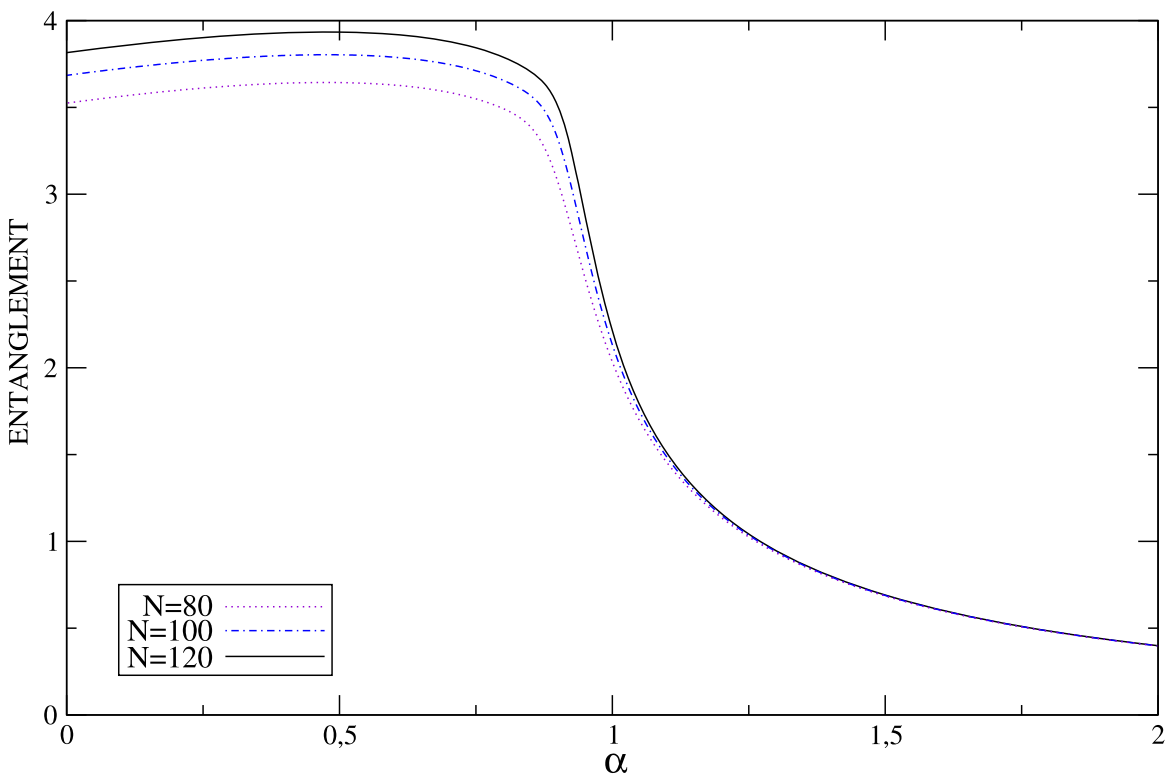

Fig. 3.2. Entanglement entropy of the ground state as a function of $\alpha$ for different values of the total number of atoms $N$. The entanglement entropy exhibits a sudden decrease close to the critical point $\alpha_{C}=1$ that becomes more pronounced as $N$ increases. 


\section{Energy gap}

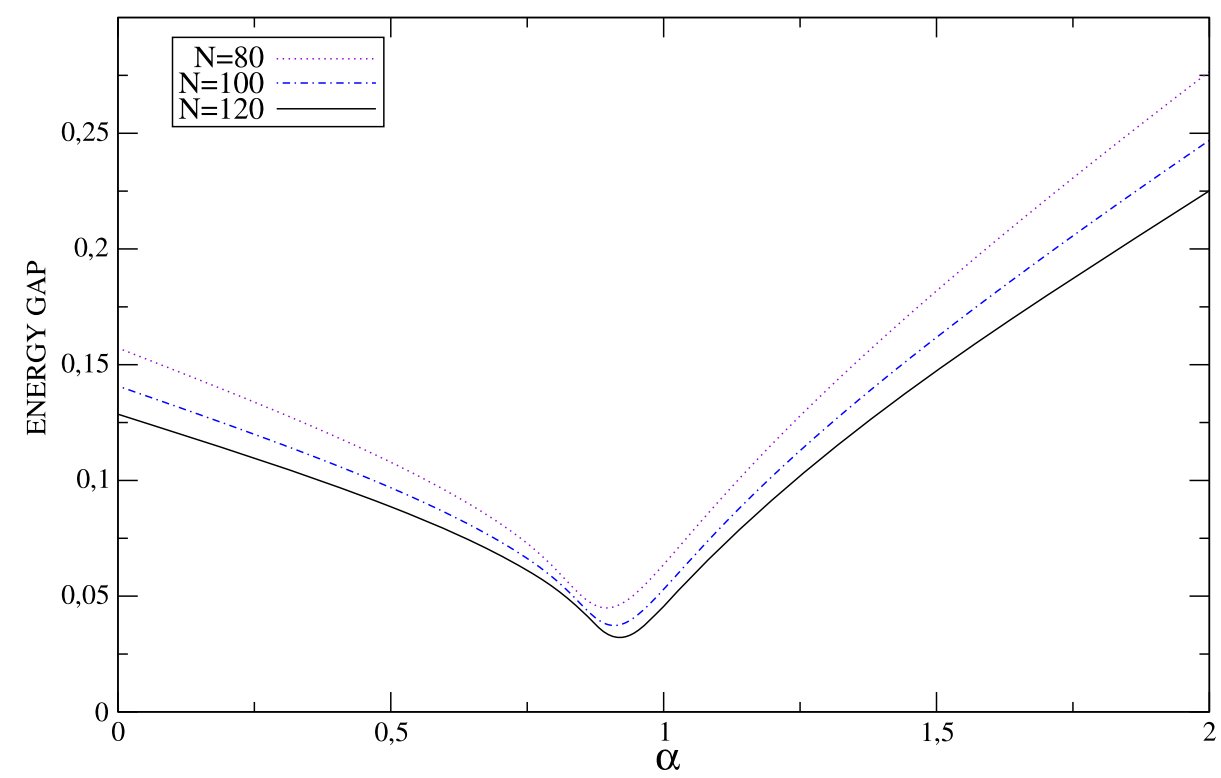

Fig. 3.3. Energy gap of the ground state as a function of $\alpha$ for different values of the total number of atoms $N$. As $N$ increases the minimum of the energy gap moves to the critical point $\alpha_{C}=1$.

\section{Fidelity}

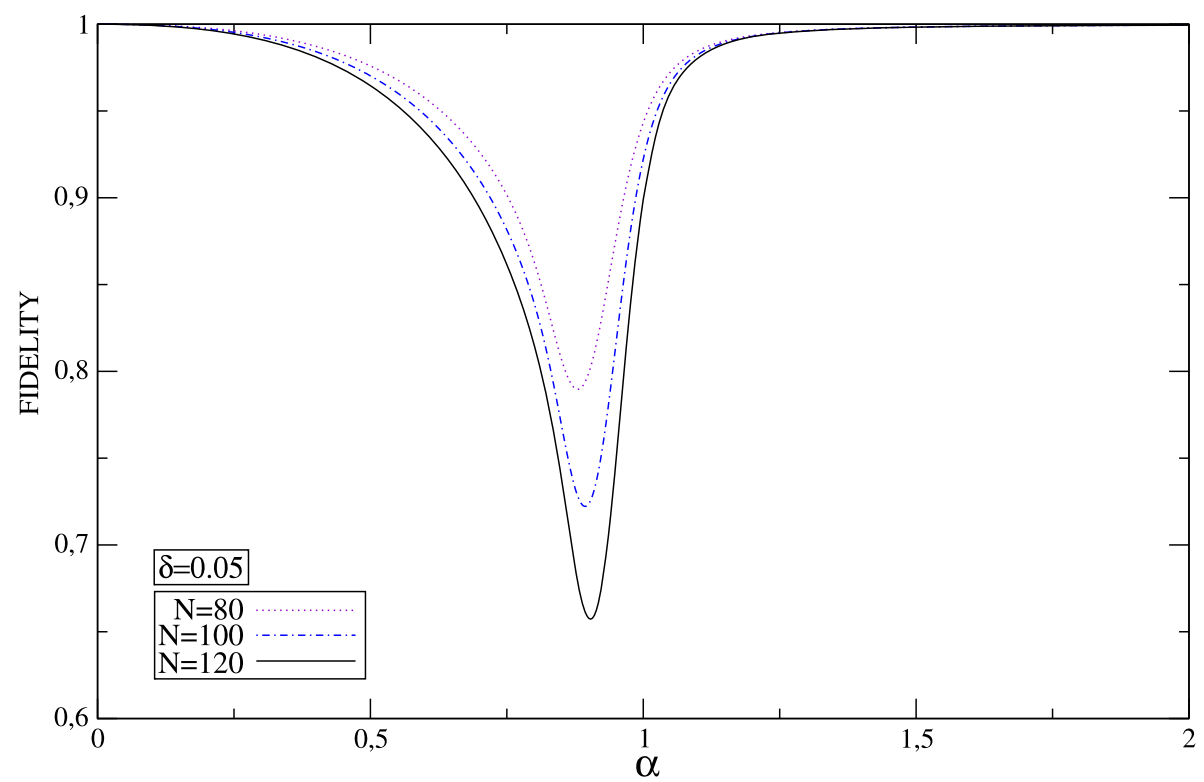

Fig. 3.4. Fidelity of the ground state as a function of $\alpha$ for different values of the total number of atoms $N$. As $N$ increases the minimum moves to the critical point $\alpha_{C}=1$. 


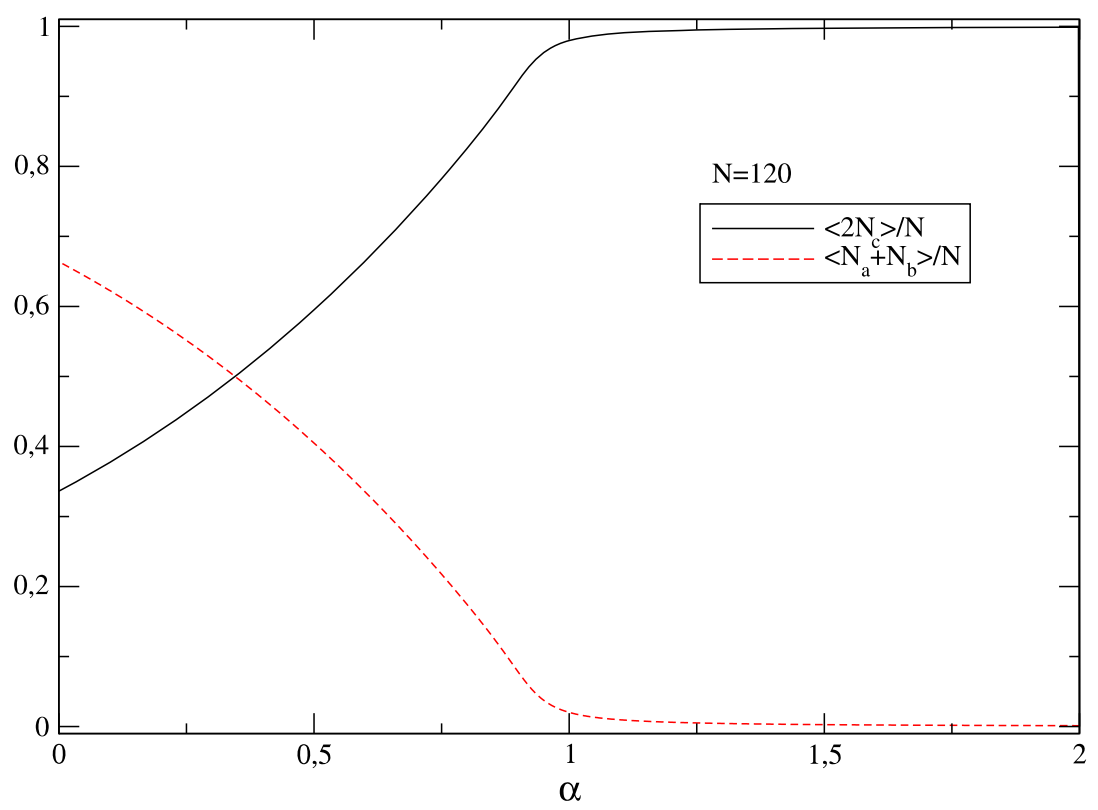

Fig. 3.5. Normalized ground state expectation value of the number of atoms and molecules in the condensate as a function of the parameter $\alpha$. We can observe a sharp transition close to the critical point $\alpha_{C}=1$.

\section{4. $B A E$ and $Q P T$}

Again, we find an agreement between the point $\alpha_{C}=1$ where the behavior of the solutions of the BAE sharply changes and the critical point obtained from the other techniques. So in the same way as for the Bose-Hubbard model, we are led to the conclusion that the solutions of the BAE can also be used as an alternative method for the identification of QPT.

We can see in Fig. 3.5 the correlation between the saturation of the number of molecules and the critical point $\alpha_{C}=1$. We note that the ground state expectation value of the normalized number of molecules increases almost linearly with increasing value of $\alpha$. When the normalized number of molecules reaches the saturation point we identify the critical point of the system. Therefore, this point can be interpreted as the critical value for which there are just molecules in the system.

\section{Summary}

The main purpose of this study was to demonstrate how the presence of very significant physical phenomena can also be inferred from the Bethe ansatz structure of a given integrable model. To illustrate this idea, we investigated the two-site Bose-Hubbard model and the hetero-atomic molecular Bose-Einstein condensate, two well known exactly solvable models, and explored their physical and mathematical properties. We began with a classical analysis for each of models exploiting the fixed point structure and making explicit the presence of bifurcation points for critical values of the relevant parameters. We then presented their energy eigenvalues by means of the quantum inverse scattering method. The BAEs thus obtained are quite involved and, apart from some limiting situations, it is virtually unfeasible to obtain an analytical solution. Never- 
theless the structure of the BAEs for each model allows the possibility of obtaining well behaved numerical solutions.

The structure of these solutions, although very different for both models, present a peculiar behavior when some parameters of the Hamiltonian are varied, indicating that the ground state solutions of the BAEs are reflecting some change in the system energy spectrum behavior. It is known that the models in question may experience a QPT and the critical parameters are well known. So, we compared our results coming from the critical points indicated by the study of the solution of the BAEs with other methods. We have performed studies of the entanglement, energy gap and fidelity for both models and the trend unveiled by these methods is highly compatible with the ground state results coming from the analysis of the solutions of the BAEs.

We have found that the quantum phase transitions for the two-site Bose-Hubbard model and for the hetero-atomic molecular Bose-Einstein condensate have very different interpretations. For the first model the transition reflects a separation between a delocalized and a localized phase, while for the second model the transition occurs when there is a saturation of molecules in the system. In particular, for the two-site Bose-Hubbard model in the delocalized phase the roots distribution is largely independent of the coupling, while in the localized phase the roots lie on different arcs. In the case of the hetero-atomic molecular Bose-Einstein condensate model all the roots are always on the positive real axis: in the phase with coexistence of atoms and molecules the roots distribution begins exactly at the origin, while in the phase where there are only molecules all roots are away from the origin. Correspondingly the profiles for both models when investigating the entanglement, energy gap and the fidelity are quite different as can be seen directly by comparing Figs. 2.5 and 3.2, for the entanglement, Figs. 2.6 and 3.3, for the energy gap, as well as Figs. 2.7 and 3.4, for the fidelity. This suggests that the quantum phase transition for each model is intrinsically different.

The models that we have analyzed were chosen for their simplicity as well as their physical relevance in the BEC context. We foresee the possibility of applying this kind of analysis in many different integrable models and this could possibly lead to some grouping according to the geometrical patterns formed by the roots such as arcs and lines, or eventually closed curves in other situations. Our approach will be particularly convenient in those cases where the exact diagonalization of the model is demanding or when the standard methods used to identify a QPT are not easily implemented. In any case we firmly believe that the behavior shown by the solutions of the BAE can be used as an alternative method to identify the presence of a QPT.

\section{Acknowledgements}

A.F. thanks J. Links for useful discussions. I. Roditi acknowledges FAPERJ (Fundação de Amparo à Pesquisa do Estado do Rio de Janeiro). The authors also acknowledge support from CNPq (Conselho Nacional de Desenvolvimento Científico e Tecnológico).

\section{References}

[1] M. Greiner, O. Mandel, T. Esslinger, T.W. Hänsch, I. Bloch, Quantum phase transition from a superfluid to a Mott insulator in a gas of ultracold atoms, Nature 415 (2002) 39-44.

[2] J. Links, H.-Q. Zhou, R.H. McKenzie, M.D. Gould, Algebraic Bethe ansatz method for the exact calculation of energy spectra and form factors: applications to models of Bose-Einstein condensates and metallic nanograins, J. Phys. A 36 (2003) R63.

[3] H.-Q. Zhou, J. Links, R.H. McKenzie, X.-W. Guan, Exact results for a tunnel-coupled pair of trapped Bose-Einstein condensates, J. Phys. A 36 (2003) L113. 
[4] J. Dukelsky, G. Dussel, C. Esebbag, S. Pittel, Exactly-solvable models for atom-molecule Hamiltonians, Phys. Rev. Lett. 93 (2004) 050403.

[5] G. Ortiz, R. Somma, J. Dukelsky, S. Rombouls, Exactly-solvable models derived from a generalized Gaudin algebra, Nucl. Phys. B 707 (2005) 421.

[6] A. Kundu, Yang-Baxter algebra and generation of quantum integrable models, Theor. Math. Phys. 151 (2007) 831

[7] A. Foerster, E. Ragoucy, Exactly solvable models in atomic and molecular physics, Nucl. Phys. B 777 (2007) 373.

[8] M. Héritier, In search of exact solutions, Nature 414 (2001) 31.

[9] M.T. Batchelor, The Bethe ansatz after 75 years, Phys. Today 60 (2007) 36.

[10] S. Sachdev, Quantum Phase Transitions, 2nd ed., Cambridge University Press, 2011.

[11] E.K. Sklyanin, L.A. Takhtadzhyan, L.D. Faddeev, Quantum inverse problem method I, Theor. Math. Phys. 40 (1979) 688-706.

[12] L.D. Faddeev, How algebraic Bethe ansatz works for integrable model, 1996; arXiv:hep-th/9605187v1.

[13] P.P. Kulish, E.K. Sklyanin, Quantum inverse scattering method and the Heisenberg ferromagnet, Phys. Lett. A 70 (1979) 461.

[14] V.E. Korepin, N. Bogoliubov, G. Izergin, Quantum Inverse Scattering Method and Correlation Functions, Cambridge University Press, 1993.

[15] F. Iachello, N.V. Zamfir, Quantum phase transitions in mesoscopic systems, Phys. Rev. Lett. 92 (2004) 212501.

[16] T. Zibold, E. Nicklas, C. Gross, M.K. Oberthaler, Classical bifurcation at the transition from Rabi to Josephson dynamics, Phys. Rev. Lett. 105 (2010) 204101.

[17] N. Bar-Gill, C. Gross, I. Mazets, M. Oberthaler, G. Kurizki, Einstein-Podolsky-Rosen correlations of ultracold atomic gases, Phys. Rev. Lett. 106 (2011) 120404.

[18] Q.Y. He, M.D. Reid, T.G. Vaughan, C. Gross, M. Oberthaler, P.D. Drummond, Einstein-Podolsky-Rosen entanglement strategies in two-well Bose-Einstein condensates, Phys. Rev. Lett. 106 (2011) 120405.

[19] J.A. Leggett, Bose-Einstein condensation in the alkali gases: Some fundamental concepts, Rev. Mod. Phys. 73 (2001) 307-356.

[20] A.P. Hines, R.H. McKenzie, G.J. Milburn, Entanglement of two-mode Bose-Einstein condensates, Phys. Rev. A 67 (2003) 013609.

[21] A.P. Tonel, J. Links, A. Foerster, Quantum dynamics of a model for two Josephson-coupled Bose-Einstein condensates, J. Phys. A 38 (2005) 1235.

[22] M. Albiez, R. Gati, J. Fölling, S. Hunsmann, M. Cristiani, M. Oberthaler, Direct observation of tunneling and nonlinear self-trapping in a single bosonic Josephson junction, Phys. Rev. Lett. 95 (2005) 010402.

[23] F. Pan, J.P. Draayer, Quantum critical behavior of two coupled Bose-Einstein condensates, Phys. Lett. A 339 (2005) 403.

[24] S. Raghavan, A. Smerzi, S. Fantoni, S.R. Shenoy, Coherent oscillations between two weakly coupled Bose-Einstein condensates: Josephson effects and $\pi$ oscillations and macroscopic quantum self-trapping, Phys. Rev. A 59 (1999) 620-633.

[25] J. Links, A. Foerster, A.P. Tonel, G. Santos, The two-site Bose-Hubbard model, Ann. Henri Poincare 7 (2006) 1591-1600.

[26] A.P. Hines, R.H. McKenzie, G.J. Milburn, Quantum entanglement and fixed-point bifurcations, Phys. Rev. A 71 (2005) 042303.

[27] S. Schneider, G.J. Milburn, Entanglement in the steady state of a collective-angular-momentum (Dicke) model, Phys. Rev. A 65 (2002) 042107.

[28] J. Links, K.E. Hibberd, Bethe ansatz solutions of the Bose-Hubbard dimer, SIGMA 2 (2006) 095.

[29] J. Links, S.-Y. Zhao, A Bethe ansatz study of the ground state energy for the repulsive Bose-Hubbard dimer, J. Stat. Mech. Theor. Exp. 2009 (2009) P03013.

[30] J.M. Román, G. Sierra, J. Dukelsky, Large- $N$ limit of the exactly solvable BCS model: Analytics versus numerics, Nucl. Phys. B 634 (2002) 483-510.

[31] M. Ibañez, J. Links, G. Sierra, S.-Y. Zhao, Exactly solvable pairing model for superconductors with $\left(p_{x}+i p_{y}\right)$-wave symmetry, Phys. Rev. B 79 (2009) 180501

[32] S.-J. Gu, S.-S. Deng, Y.-Q. Li, H.-Q. Lin, Entanglement and quantum phase transition in the extended Hubbard model, Phys. Rev. Lett. 93 (2004) 086402.

[33] M.A. Nielsen, I.L. Chuang, Quantum Computation and Quantum Information, Cambridge University Press, 2000.

[34] P. Buonsante, A. Vezzani, Ground-state fidelity and bipartite entanglement in the Bose-Hubbard model, Phys. Rev. Lett. 98 (2007) 110601.

[35] C. Weber, G. Barontini, J. Catani, G. Thalhammer, M. Inguscio, F. Minardi, Association of ultracold double-species bosonic molecules, Phys. Rev. A 78 (2008) 061601. 
[36] C. Haimberger, J. Kleinert, M. Bhattacharya, N.P. Bigelow, Formation and detection of ultracold ground-state polar molecules, Phys. Rev. A 70 (2004) 021402.

[37] L.D. Carr, D. DeMille, R.V. Krems, J. Ye, Cold and ultracold molecules: Science and technology and applications, New J. Phys. 11 (2009) 055049.

[38] D.F. Walls, R. Barakat, Quantum-mechanical amplification and frequency conversion with a trilinear Hamiltonian, Phys. Rev. A 1 (1970) 446-453.

[39] M. Duncan, A. Foerster, J. Links, E. Mattei, N. Oelkers, A. Tonel, Emergent quantum phases in a heteronuclear molecular Bose-Einstein condensate model, Nucl. Phys. B 767 (2007) 227.

[40] L. Zhou, W. Zhang, H.Y.L.L. Jiang, H. Pu, Properties of a coupled two species atom-heteronuclear molecule condensate, Phys. Rev. A 75 (2007) 043603.

[41] L. Zhou, J. Qian, H. Pu, W. Zhang, H.Y. Ling, Phase separation in two-species atomic Bose-Einstein condensate with interspecies Feshbach resonance, Phys. Rev. A 78 (2008) 053612. 